\title{
Théologiques
}

Théologiques

\section{Q Florilegium}

\section{Robert David}

Volume 24, numéro 2, 2016

Construction des identités religieuses : hommage à Jean Duhaime

URI : https://id.erudit.org/iderudit/1050509ar

DOI : https://doi.org/10.7202/1050509ar

Aller au sommaire du numéro

Éditeur(s)

Faculté de théologie et de sciences des religions, Université de Montréal

ISSN

1188-7109 (imprimé)

1492-1413 (numérique)

Découvrir la revue

Citer ce document

David, R. (2016). 12Q Florilegium. Théologiques, 24(2), 241-253.

https://doi.org/10.7202/1050509ar

Ce document est protégé par la loi sur le droit d'auteur. L'utilisation des services d'Érudit (y compris la reproduction) est assujettie à sa politique d'utilisation que vous pouvez consulter en ligne.

https://apropos.erudit.org/fr/usagers/politique-dutilisation/
Cet article est diffusé et préservé par Érudit.

Érudit est un consortium interuniversitaire sans but lucratif composé de l’Université de Montréal, l'Université Laval et l'Université du Québec à Montréal. Il a pour mission la promotion et la valorisation de la recherche. https://www.erudit.org/fr/ 


\title{
$12 Q$ Florilegium ${ }^{1}$
}

\author{
Robert DAviD" \\ Exégèse vétérotestamentaire \\ Université de Montréal
}

En ce 11 septembre 2013, date qui résonne étrangement à nos oreilles depuis 2001, je voudrais vous faire part d'une nouvelle extraordinaire, qui associera dorénavant cette date à une toute nouvelle réalité, plus positive et plus stimulante.

Le 28 juillet 1999, vers 10 h30 du matin, alors que j'arpentais les rochers marneux des abords de la mer Morte, à environ 600 mètres au sud des installations communautaires de Qumrân, je décidai de m'assoir pour admirer le paysage qui s'offrait à moi. Au moment de repartir, une trentaine de minutes plus tard, je cherchai à reprendre mon bagage. Par inadvertance, ma main fit rouler un caillou. Une mince lanière de cuir apparut alors, qui dépassait à peine du sol, là même où se trouvait le caillou quelques instants plus tôt. La couleur de la lanière se confondait avec celle des roches environnantes. Excité, incrédule, je décidai de dégager la lanière. En creusant un peu, je retirai quelques pierres et révélai une petite cavité d'environ 2 mètres cube. Je pensai que le soleil qui plombait sur ma tête depuis quelques heures me causait des hallucinations lorsque je mis la main sur un artéfact exceptionnel, dont j'ai gardé l'existence secrète jusqu'à aujourd'hui. Je glissai l'objet dans mon sac à dos, et décidai de le rapporter à la maison, espérant que les autorités douanières israéliennes n'allaient pas mettre la main dessus à l'aéroport Ben Gourion. Depuis maintenant 14 ans, je suis en possession de cet artéfact. J'ai travaillé sur cette découverte, seul, fébrile et anxieux, sans jamais en aviser le département des Antiquités d'Israël, ni Emanuel Tov, et encore moins Hershel Shanks.

1. Cet article reprend littéralement le texte de l'hommage rendu au professeur Jean Duhaime lors d'une soirée qui fut consacrée à souligner son départ à la retraite.

* Robert David est professeur honoraire de la Faculté de théologie et de sciences des religions de l'Université de Montréal (connue désormais sous le titre d'Institut d'études religieuses).

C Revue Théologiques 2016. Tout droit réservé. 
Aujourd'hui, j'ai décidé de rendre public les résultats de mes travaux, confiant que vous saurez pardonner mon écart de conduite, ma faute éthique et mon égoïsme professionnel. Je vous présente donc, en première mondiale, l'editio princeps d'un nouveau manuscrit de la mer Morte auquel, après l'avoir déchiffré et commenté, j'ai donné le nom provisoire de 12 QFlorilège.

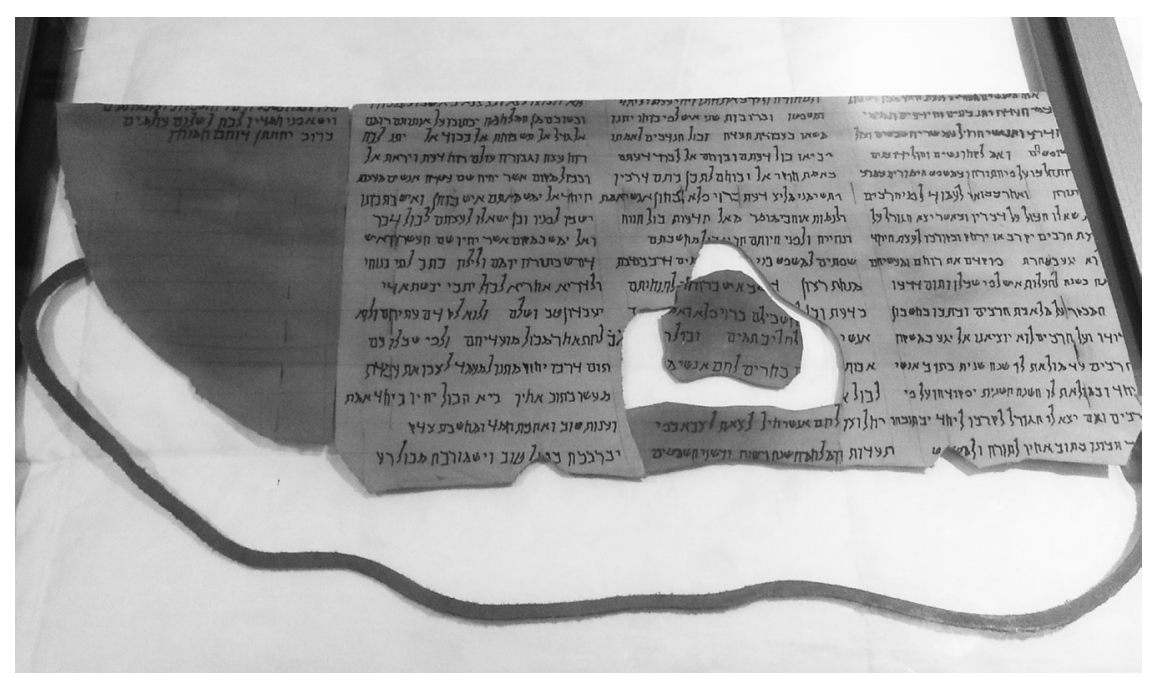

\section{Description de $12 \mathrm{Q}$ Florilegium}

Vous me permettrez d'abord de vous décrire brièvement l'objet de ma découverte:

- Largeur totale: $46 \mathrm{~cm}$; hauteur: $19 \mathrm{~cm}$;

- 4 fragments de cuir, dont 2 petits associés au centre et au bas de la colonne II + 1 lanière de cuir;

- 2 peaux différentes ont été utilisées (couleur différente de la colonne IV), mais le lignage semble avoir été fait en liant les deux peaux puisque la jonction des lignes se fait parfaitement de la peau plus pâle à la plus foncée;

- 4 colonnes pré-lignées;

- Largeur des colonnes: $10 \mathrm{~cm}$ sauf la première $(9,8 \mathrm{~cm})$ à laquelle il manque la $1^{\text {re }}$ lettre de chaque ligne;

- Marges entre les colonnes: $1 \mathrm{~cm}$;

- Hauteur des lignes: $1 \mathrm{~cm}$; 
- Nombre de lignes par colonne: 16;

- Environ 35 lettres par lignes;

- Marge supérieure: incomplète (il manque la portion supérieure des lettres des colonnes II-IV);

- La colonne IV est lignée mais elle ne possède que 3 lignes de texte;

- Écriture: post-hérodienne;

- Présence de coupures verticales dans le sens de la hauteur et de la largeur ( $6^{\mathrm{e}}$ ligne) dans le cuir à intervalle régulier. Les coupures sur la hauteur ont pu servir à lier les peaux ensemble grâce à la lanière retrouvée, qui a la même largeur que les coupures. Je m'interroge encore sur l'utilité des coupures sur le sens de la largeur.

\section{Quelques particularités de 12 QFlor.}

\section{Colonne I}

- On peut penser que deux copistes distincts ont travaillé sur ce manuscrit, l'écriture des trois premières lignes de la colonne I étant plus grossière que celle du reste du manuscrit.

- Il manque la première lettre de toutes les lignes sauf lignes 1 et 11 (vacat au début).

- Le copiste a oublié une lettre $(\mathrm{H})$ dans le premier mot de la ligne 4, lettre qu'il a suscrite.

- Le copiste a utilisé la marge entre les colonnes I et II pour terminer des mots aux lignes 9 et 15 .

- La présence de vacat marque les limites des citations utilisées dans ce florilège.

\section{Colonne II}

- On peut voir à la colonne II ligne 12 que le copiste a marqué deux lettres (Aleph et Beth) qui doivent être effacées. Il a sans doute été distrait puisque les deux lettres, qui se trouvent dans l'entre colonnes, sont reprises au début de la ligne 13 .

- Les fragments B et C s'insèrent dans la grande lacune centrale.

- La ligne 14 est presque totalement absente (manque 27 lettres) mais le contenu peut être reconstitué grâce à la ligne 15 qui continu le texte amorcé à la ligne $12 \mathrm{~b}$.

\section{Colonne III}

- On ne dispose que du bas des lettres de la ligne 1

- Colonne complète 


\section{Colonne IV}

- Toute la colonne est lignée, mais elle n'a que 3 lignes de texte, ce qui prouve que nous avons la fin du manuscrit.

- Nous n'avons que la base des lettres de la ligne 1, mais le texte amorcé à la fin de colonne III 16 et qui se continue en IV 2 permet de reconstituer ces lettres.

- Comme nous le verrons plus tard, la traduction de la ligne 3 nous a donné la clef d'interprétation du manuscrit.

\section{Traduction et commentaires}

Ces quelques remarques complétées, il me fait plaisir de vous présenter la traduction de ce manuscrit, ainsi que le commentaire qui accompagne chacune des strophes. Le travail que j'ai fait dans les archives de la Communauté m'aura permis d'arriver à une interprétation que je crois juste et fidèle, mais qui pourra être parfaite au fur et à mesure que des chercheurs pourront se pencher sur 12QFlor à partir de maintenant.

\subsection{Q Flor I}

Voici les personnes convoquées pour le Conseil de la Communauté: tous les sages de la Congrégation et les intelligents et les connaissants, parfaits de conduite et hommes valides, ainsi que les chefs des tribus et tous leurs juges et leurs intendants. (1QSa I 27- 29)

Ceci concerne les membres réunis pour témoigner leur appréciation au Maître de l'enseignement. Les sages de la Congrégation, ce sont les dignitaires; les intelligents ce sont ses collègues; les connaissants parfaits de conduite, ce sont les membres de sa famille; les hommes valides, ce sont ceux qu'il a accompagnés tout au long de sa carrière; les chefs des tribus, juges et intendants, ce sont les amis, connaissances, et toute autre personne venue témoigner de son admiration et de son attachement au Maître.

Et s'ils prennent femme et engendrent des enfants, ils marcheront dans

l'obéissance à la Loi et selon l'ordonnance relative aux engagements, selon la règle de la Loi. (Écrit de Damas VII 6-8)

Ceci concerne l'épouse fidèle et constante du Maître, ainsi que ses deux enfants qui, dans les jours de Lumière comme dans les jours de Ténèbres, ont été aux côtés du Maître, l'ont supporté et, parfois, malgré eux et elle, se sont retrouvés à devoir vivre selon les lois et les décrets 
qu'oblige une vie consacrée à la Communauté. Ils ont été, sans le vouloir peut-être, des membres à part entière de la Communauté via leur soutien indéfectible et patient au Maître. Puisse la Congrégation leur en être éternellement reconnaissante.

Quand il viendra pour se présenter devant les Nombreux, ils délibéreront, eux tous, sur son cas; et, selon ce que le sort prononcera, d'après la décision des Nombreux, il s'approchera ou bien il s'éloignera. (1QS VI 15-16)

Ceci concerne le premier engagement du Maître dans la Communauté en avril 1976, mais surtout en juin 1977. Les Nombreux, ce sont les membres de l'Assemblée et du Conseil qui se sont interrogés sur les qualifications antérieures du Maître ${ }^{2}$, ses aptitudes théologiques, la valeur de sa formation. Dans la sagesse de leurs délibérations, ils auront vu le potentiel du Maître et l'auront admis dans la Communauté, d'abord à demi-temps (1977-1979), puis comme substitut à l'Enseignant Martucci (1979-1982), le faisant ainsi passer à plein temps dans la Communauté.

L'on examinera leur esprit et leurs œuvres année après année, de façon à promouvoir chacun selon son intelligence et la perfection de sa conduite. (1QS V 24)

Ceci concerne l'évaluation annuelle que le Maître a dû produire tout au long de sa présence au sein de la Communauté. Les rapports déposés par le Maître dans les archives de la Communauté permettent de dresser un portrait assez détaillé de ses activités, de ses intérêts, de ses enseignements, de ses recherches, de ses écrits et de ses innombrables contributions au sein de la Communauté, mais également pour la Congrégation et l'Assemblée de sa Qahal.

Il ne touchera pas au banquet des Nombreux avant qu'il n'ait achevé une seconde année au milieu des membres de la Communauté. Puis, quand il aura achevé la seconde année, on l'examinera: d'après la décision des Nombreux, et si le sort prononce qu'il s'approche de la Communauté, on l'inscrira régulièrement à son rang parmi ses frères... il pourra donner son avis à la Communauté, ainsi que sa sentence. (1QS VI 20-23)

Ceci concerne les diverses étapes des promotions du Maître au sein de la Communauté. Le banquet des Nombreux, ce sont les diverses instances

2. Licence de Sciences Religieuses à l'Université de Montréal en 1971, M.A études bibliques en 1974 et Étudiant diplômé de l'École biblique et archéologique française de Jérusalem en 1976. 
de la Communauté et de la Grande Communauté. La seconde année concerne l'étape menant à l'agrégation et à la permanence, étape que le Maître a franchie avec brio en 1982. L'achèvement de la seconde année concerne le passage à la titularisation, en 1989, étape marquante qui a consacré le Maître parmi les grands de la Congrégation, la Communauté ayant reconnu l'apport remarquable et remarqué du Maître dans le domaine de ses recherches et dans son rayonnement au sein des nations.

\section{$3.212 Q$ Flor II}

Et, quand une personne aura de nombreuses années, c'est à la mesure de sa force qu'on lui confiera une charge au service de la Communauté. (1QSa I 19)

Ceci concerne le passage progressif du Maître à la retraite, passage amorcé en 2010 et qui s'est terminé en août 2013. Malgré son âge avancé et les recommandations de l'Instruction, le Maître n'a jamais vraiment allégé son travail, consacrant toujours de nombreuses heures à l'étude de la Loi et des décrets, aux Règles de la guerre et à la compréhension des deux Esprits. Sa présence au sein de l'Assemblée de la Communauté s'est toutefois faite plus discrète, le Maître sentant qu'il devait dorénavant laisser les nouveaux membres admis au sein de la Communauté diriger les destinées de cette dernière.

Et tous les volontaires attachés à Sa vérité apporteront toute leur intelligence et toutes leurs forces afin de purifier leur intelligence dans la vérité des préceptes de Dieu et d'ordonner leurs forces selon la perfection de Ses voies (1QS I 11-13)

Ceci concerne les divers enseignements du Maître tout au long de ses jours dans la Communauté. Il a abordé 26 thèmes de cours différents, offerts par une centaine de prestations. S'il a souvent enseigné l'introduction aux Écritures (19 fois), l'étude de la Torah (12 fois) et des Psaumes (18 fois), il s'est également montré intéressé par les questions d'interprétation ( 5 fois), par le prophétisme et la sagesse ( 15 fois), les approches sociologiques (pour lesquelles il s'est formé entre 1992-1996 en décrochant une Maîtrise en sociologie avec une note parfaite de $A+4,3)$, ainsi que divers thèmes pour lesquelles ses compétences et intérêts ont été sollicités. Il s'est même commis dans un cours portant sur l'Initiation au Nouveau Testament! Il aura réussi, au fil des ans et à force de persévérance et d'acharnement, à intégrer des perspectives qumraniennes dans ses offres de cours, liant de cette façon sa passion première avec les obligations de la 
formation générale. Toujours, les disciples ont été enchantés des prestations du Maître et ont évalué très positivement ses enseignements, reconnaissant sans contredit la profondeur et l'étendue de ses savoirs, ainsi que la maîtrise parfaite de ses contenus. Il a contribué à purifier leur intelligence dans la vérité et à en faire des membres de la Congrégation dont les forces furent ordonnées selon la perfection des Voies de Dieu.

$\mathrm{Tu}$ as fait de moi un interprète (plein) de Connaissance concernant les Mystères merveilleux, pour éprouver les hommes de vérité et pour mettre à l'épreuve ceux qui aiment l'instruction. (Hodayot X 15-16a)

Ceci concerne les divers sujets de recherche qui ont passionné le Maître tout au long de sa carrière, particulièrement celui de la communauté des bords de la mer Morte. Amorcée avant son entrée dans la Communauté par des études portant sur l'Instruction des deux Esprits (1QS III 13 - IV 26) et sur les Interprétations dualistes dans les textes de Qumrân, ses recherches ont beaucoup porté sur le Rouleau de la guerre, dont il est devenu le spécialiste reconnu internationalement, ainsi que sur la Règle de la communauté. Bien que ces deux sujets aient particulièrement retenu son attention au cours des 40 dernières années, le Maître ne s'est pas limité à cela. En font foi les divers sujets qu'il a traités en dehors des études qumraniennes: le paradis, genèse et évolution; les sociétés devant le pluralisme religieux; les approches sociologiques de la Bible; la souffrance dans les Psaumes; David et les Psaumes; anges et démons dans la littérature intertestamentaire, et d'autres encore. Pour chacun de ces sujets, le Maître a su mener ses recherches avec rigueur et intégrité, patience et détermination, ouvrant parfois des chantiers encore peu explorés, comme celui de l'apport des approches sociologiques dans l'étude des textes de Qumrân, et ceux de la Bible

Du Dieu des Connaissances provient tout ce qui est et sera; et avant que les êtres ne fussent, Il a établi tout leur plan. (1QS III 15)

Ceci concerne les antécédents du Maître, avant son entrée dans la Communauté. S’il est devenu le Maître incontesté des écrits portant sur les Fils de Lumière et sur les Fils des Ténèbres, il y était déjà prédestiné par ses activités comme responsable des éclairages avec des troupes de théâtre. Le Dieu des connaissances l'y avait donc préparé, à son insu, introduisant dans son cœur et son esprit, les ingrédients nécessaires à sa destinée remarquable.

L'offrande des lèvres dans le respect du droit, sera comme une agréable odeur de justice, et la perfection de la voie sera comme le don volontaire d'une oblation délectable. (1QS IX 5) 
S'il fut un enseignant aimé et apprécié, le Maître n'en a pas moins été un conférencier recherché et prisé. L'offrande de ses lèvres concerne les dizaines et dizaines de conférences scientifiques soigneusement préparées, toujours très bien documentées et présentées avec enthousiasme et compétence, comme une oblation délectable, dans divers congrès nationaux et internationaux. S'ajoutent à cela les dizaines de prestations de conférences publiques, d'entrevues et de communications, dons volontaires portant sur les manuscrits de Qumrân, mais également sur le dialogue judéo-chrétien et sur les nouveaux mouvements religieux, entre autres, toujours dans le respect et la perfection de la voie de chacun.

Chacun selon son esprit, il les guidera dans la Connaissance, et pareillement il les instruira des Mystères merveilleux et véridiques au milieu des membres de la Communauté, pour qu'ils marchent dans la perfection (1QS IX 18-19)

Ceci concerne les nombreuses directions de disciples, que le Maître a su guider dans les méandres de la recherche, sur des sujets aussi divers que l'Inspiration des Écritures, les 4 sens de l'Écriture, l'éducation de la foi des adultes, des sujets bibliques associés aux Psaumes ou à la Sagesse, pour ne nommer que quelques thèmes. Il a guidé une trentaine de personnes à la maîtrise et une quinzaine au doctorat, en plus de la cinquantaine de jury auxquels il a participé à titre de membre, de président ou de directeur. Son plus grand regret, sans doute, aura été de ne pas avoir pu diriger plus d'étudiants dans des sujets qui concernaient directement ses champs de recherche privilégiés, soit les études qumraniennes pour lesquelles 3 doctorats et 3 maîtrises ont été menés sous sa gouverne, sujets qui touchaient le document de Damas, les hymnes de Qumrân, les anges et les démons, la cérémonie d'alliance, Melkisédek et le messianisme. Dans toutes ces directions, le Maître aura su emmener ses protégés à développer leur sens critique, à parfaire leurs connaissances et leurs aptitudes méthodologiques, et à produire des recherches à la fine pointe des domaines étudiés. Au cours de ses années dans la Communauté, il aura su distiller son souci du détail, du travail bien fait et de la probité intellectuelle.

Et tous les chefs de famille de la Congrégation se choisiront des combattants pour tous les territoires des nations... Ils mobiliseront pour eux les hommes valides pour qu'ils partent conformément aux prescriptions de la guerre, année par année; mais dans les années de moratoires, ils ne mobiliseront personne pour partir à l'armée, car c'est un sabbat de repos. (1QM II 7b-9a)

Ceci concerne les nombreux déplacements que le Maître a menés au fil des ans pour aller diffuser et partager sa Sagesse et ses Connaissances. 
Les territoires des nations ce sont les cités d'Atlanta, de Chicago, de Gröningen, de Paris, d'Oxford, de Jérusalem, de Louvain, de Mulhouse, de Strasbourg, de Vancouver, de Toronto, d'Ottawa, de Québec et de Montréal. Durant toutes ces années, il a ainsi participé à plus d'une quarantaine de congrès scientifiques, prononcé des dizaines de conférences de haut niveau, conquis, selon les prescriptions de la guerre intellectuelle, les esprits et les cœurs de ses auditeurs à qui il a transmis sa passion pour la sociologie, le dialogue interreligieux, entre autres, mais surtout, surtout, pour ses recherches sur Qumrân. À ce chapitre, il est dorénavant reconnu comme une sommité en la matière, principalement pour ses contributions à une meilleure compréhension de la Règle de la guerre, de la Règle de la Communauté, et du dualisme.

\section{$3.312 Q$ Flor III}

Et quand ils reviendront du combat, on inscrira sur leurs étendards: «Dieu est élevé », «Dieu est grand », «Éloge de Dieu», «Gloire de Dieu». (1QM IV 8)

Ceci concerne la grandeur des contributions du Maître et le jugement porté sur celles-ci au retour de ses déplacements chez les nations. Elles inscrivent ses lettres de noblesse et affirment, haut et fort, combien son travail, et sa personne, élèvent le monde vers un niveau supérieur qui fait la gloire de Dieu et font rayonner Sa grandeur sur le monde que le Maître contribue à rendre meilleur dans la connaissance et la droiture d'esprit.

Puisse-t-il te donner un esprit de Conseil et de courage éternel, un esprit de connaissance et de crainte de Dieu. (1QSb V 25)

Ceci concerne la présence du Maître dans les nombreux comités auxquels il a apporté une contribution inestimable, tant au sein de la Communauté qu'à l'intérieur de la Grande Communauté. Par sa sagesse et ses conseils précieux, il a apporté des solutions et des recommandations tant à l'Assemblée qu'au Conseil de la Communauté. Au cours de ses trois mandats à titre de vice-chef du Conseil (vice-doyen 1985-1993; 1997 2001) il a mené à bien une réforme du premier cycle et fait avancer des dossiers importants pour le positionnement de la Communauté au milieu de la Grande Communauté. Pendant plusieurs années il a également prodigué ses conseils au sein du conseil de la $\mathrm{FESP}^{3}$, en plus de siéger au comité de la bibliothèque de la Grande Communauté. Le Maître connais-

3. Faculté des études supérieures et postdoctorales. 
sait à peu près tous les rouages et les mécanismes qui permettent à la Communauté de fonctionner le plus harmonieusement possible. Son sens de l'organisation et sa connaissance des dossiers ont été des valeurs sûres pour l'ensemble des collègues qui ont pu en bénéficier pendant toutes ces années.

Et en tout lieu où il y aura dix personnes du Conseil de la Communauté, qu'il ne manque pas parmi eux un homme qui soit prêtre. Et que, chacun selon son rang, ils s'asseyent devant lui; et que, selon le même ordre, on leur demande leur avis en toute chose. (1QS VI 3-4)

Ceci concerne le rôle de Chef du Conseil (doyen) que le Maître a exercé pendant 4 années (2005-2009). Le Maître aura dû user de toute son habileté et de sa sagesse pour diriger la Communauté pendant cette période difficile, alors que les disciples se faisaient plus rares et que des collègues quittaient la Communauté, l'âge avancé les y obligeant. Le Maître a dû faire preuve de doigté et d'inventivité pour bien gérer la Communauté et s'assurer de sa survie durant la période sombre qui a précédé celle de la grande réforme. Les membres de la Communauté lui sont redevables d'avoir su mener la barque à bon port malgré la tempête qui grondait.

Et qu'il ne manque pas, dans le lieu où seront les dix, un homme qui étudie la Loi jour et nuit, constamment. (1QS VI 6)

Ceci concerne le travail tenace du Maître qui n'a pas compté ses heures au service de la Connaissance et de l'intelligence de la Loi, des préceptes et des décrets. Bien qu'il ait parfois inquiété les membres de la Communauté par la pâleur de son teint et l'étirement de ses traits, le Maître a toujours montré une constance et un acharnement dans le travail, allongeant le jour, diminuant ses nuits. Le combat de ce Fils de la Lumière contre les Ténèbres aura marqué le temps de son labeur.

Il a écrit à ses fils, et aux fils de ses fils, et aux futures générations, à tous ceux qui demeurent dans la contrée sèche, afin qu'ils fassent le bien et la paix. (4Q212 II 23)

Ceci concerne les très nombreux écrits produits par le Maître tout au long de sa carrière. Ses écrits, portant en grande partie sur la communauté de Qumrân, mais aussi sur divers thèmes associés aux Écritures, à la sociocritique, au dialogue judéo-chrétien ou à l'émergence des nouveaux mouvements religieux, ont été publiés dans des collectifs ou à titre individuels. Une soixantaine d'articles scientifiques sont parus dans quelques-unes des revues les plus importantes telles que Catholic Biblical Quarterly, Journal 
for the Study of Judaism, Revue biblique, Revue de Qumrân, Religious Study Review, Henoch, Hebrew Studies, Sciences religieuses, Social Compass, Laval Théologique et philosophique, Sciences religieuses, Sciences et Esprit, pour n'en nommer que quelques-unes. À cette production de haut calibre, il faut ajouter une centaine de recensions qu'il a faites paraître dans diverses revues et sa trentaine de collaborations à des ouvrages collectifs, dont certains font aujourd'hui autorité de par les nations. La renommée du Maître et sa connaissance approfondie des textes de Qumrân, lui ont valu d'être sollicité pour produire des œuvres qui, aujourd'hui, sont des références incontournables dans le monde des études qumraniennes. La consécration du Maître est confirmée par la production de son «War Scroll », paru en 1995 dans J. H. Charlesworth The Dead Sea Scrolls 2, Louisville, Westminster/John Knox, 80-203, sa "Règle de la Communauté» (1998) dans J. H. Charlesworth The Dead Sea Scrolls, Philadelphia, ses articles "Light and Darkness ", "Determinism» et "Dualism» (2000) dans l'Encyclopedia of Dead Sea Scrolls, NewYork, Oxford University Press, son «War Texts» (2006), publié dans la collection Companion to the Qumran Scrolls 6, son «War Scroll» (2010) paru dans le Eerdmans Dictionary of Early Judaism, Grand Rapids, Eerdman et Les rouleaux de la mer Morte (2010) qu'il a fait paraître dans le Supplément au Cahiers Évangile 152, Paris, Cerf, et, plus récemment, sa coédition (avec Peter W. Flint et Kyung S. Baek) de Celebrating the Dead Sea Scrolls. A Canadian Collection (2011), Atlanta, Society of Biblical Literature ${ }^{4}$. Et le Maître travaille toujours à la préparation d'un commentaire de la Règle de la guerre, ainsi que sur un projet international de traduction critique en français des pesharim de Qumrân et des textes de sagesse. Avec constance, dans l'ensemble de ses écrits, le Maître a su faire montre de rigueur, d'originalité, de profondeur et d'un sens aigu de l'analyse et de la méthode. Assurément, les nations profiteront longtemps de ses écrits, témoins de sa vaste connaissance, de ses divers intérêts, de sa passion pour le savoir et l'étude, le travail bien fait et la diffusion au plus grand nombre du fruit de ses longues heures de labeur.

Ils ne devanceront leurs temps, ni ne seront en retard pour aucune de leurs fêtes. (1QS I 14-15)

4. Traduction en français de cet ouvrage: (2014) Célébrer les manuscrits de la mer Morte. Une perspective canadienne, Montréal, Médiaspaul. 
Ceci concerne une Règle que le Maître semble n'avoir jamais lue, ou, à tout le moins, n'avoir jamais vraiment comprise.

Dans la mesure de son intelligence, ainsi que dans la perfection de sa conduite, il fortifiera ses reins au poste qu'il occupe, afin d'exercer la fonction confiée à ses soins au milieu de ses frères (1QSa I 17-18)

Ceci concerne les très nombreuses implications du Maître en dehors de la Communauté, au service de l'Assemblée. Sa sagesse et son esprit de conseil ont été utilisés à bon escient alors que le Grand-Prêtre de son Assemblée l'a mandaté en 1999 pour représenter officiellement la Qahal auprès du groupe de dialogue judéo-chrétien, fonction qu'il a occupée pendant quelques années et qui l'a mené à collaborer avec ce groupe jusqu'à ce jour. Cela concerne aussi le souci du Maître de continuer à répandre la Connaissance dans des groupes qui n'ont pas toujours accès à la Grande Communauté, soit par le biais de ses écrits de vulgarisation, son implication dans sa Qahal auprès des jeunes adultes, ses nombreuses entrevues dans les médias, souvent en lien avec les écrits de Qumrân dont il aime partager la connaissance. Il a aussi ouvert son cœur et son esprit à des organismes de diffusion et d'information tels que le Conseil de $\mathrm{SOCABI}^{5}$ et le Centre d'Information sur les nouvelles religions. Le Maître a ainsi démontré qu'en plus de consacrer une partie importante de sa vie à la recherche et à la connaissance, il était aussi primordial de faire profiter le plus grand nombre possible des grâces du savoir et, qui sait, paver ainsi la voie à de nouvelles vocations de chercheurs au sein de la Communauté.

Car tous, ils seront dans la Communauté de vérité et de vertueuse humilité et d'affectueuse charité et de scrupuleuse justice. (1QS II 24-25)

Ceci concerne les merveilleuses qualités qui ont fait la renommée du Maître au sein de la Communauté et au-delà, dans toutes les nations. Tous ceux et celles qui l'ont côtoyé reconnaissent dans le Maître son ouverture d'esprit, sa générosité de temps et de savoir, son sens du travail bien fait et de la précision, son souci du détail, son amour de la justice et du droit, sa passion pour la Connaissance et l'intelligence, son attention aux autres, la sagesse de ses conseils, son Amour de Dieu et des siens, sa loyauté et son intégrité, sa patience et sa persévérance. Il est et restera un modèle d'équilibre entre savoir et vie concrète, intelligence et empathie. Le Maître restera une Lumière, une voie pour ceux et celles qui joindront la Communauté à

5. Société catholique de la Bible. 
l'avenir, même s'ils ne pourront plus profiter de sa présence effective et tangible.

Qu'Il te bénisse en tout bien, et qu'Il te garde de tout mal! Qu'Il illumine ton cœur par l'intelligence de vie, et qu'Il te favorise de la Connaissance éternelle!

Et qu'Il lève vers toi Sa face gracieuse pour t'accorder l'éternel bonheur. (1QS II 2-4)

Ceci concerne la bénédiction que tous et toutes souhaitent formuler pour le Maître, bénédiction qui, à elle seule, résume les souhaits les plus sincères de la Communauté à l'endroit du Maître, et à laquelle il serait inutile d'ajouter quoi que ce soit, sinon d'y joindre la voix de toute la Congrégation.

\subsection{Q Flor IV 3}

La dernière ligne du manuscrit fut pour moi une véritable révélation. Bien que j'aie eu quelques difficultés à saisir le sens du $3^{\mathrm{e}}$ mot, j'ai fini par comprendre qu'il s'agissait-là de la clef pour interpréter et commenter l'ensemble du manuscrit, ce que j'ai fait pour vous aujourd'hui.

Cette $3^{\text {e }}$ ligne se lit comme suit: "Béni (soit) Yonathan Duhem LE Maître. »

Au terme de cette présentation, et à la lumière de 12QFlorilegium IV 3, je me considère autorisé à renommer ce manuscrit de la façon suivante: 12Q Florilegium Moreh Yonatan Duhaimeniensis

Et à l'offrir au Maître en guise de modeste présent, témoignage de mon affection pour les 37 années au cours desquels j'ai pu le côtoyer, à titre d'étudiant, de collègue, de conseiller, de chercheur et surtout, d'ami.

Bonne retraite, Jean 